\title{
La localisation spatiale par effet Doppler ultrasonore
}

\author{
L. BITJOKA, A. RONCIN et L. POURCELOT
}

GIP Ultrasons, Faculté de Médecine, Laboratoire de Biophysique Médicale, Bâtiment Vialle, 2 bis bd Tonnellé, BP. 3223, 37032 Tours cedex, France

\begin{abstract}
The problem of the two-dimensional (2-D) localization of a target by the ultrasound Doppler effect is theoretically investigated, adopting the case of a system composed of one transmitter and two receivers. Accordingly the relation between the Doppler frequency shift at each receiver and the 2-D localization of the target was established. The range $R$ and the azimuth $\theta$ are derived from the maximum likelihood Doppler signal processing. The first stage consisted in performing the localization in air, only at the left or at the right side of the line-of-sight of the system.
\end{abstract}

\section{INTRODUCTION.}

La disposition géométrique du récepteur par rapport à l'émetteur d'ultrasons permet d'établir la relation entre le décalage de fréquence Doppler [1-2] et la localisation spatiale 2-D d'un réflecteur. Lorsque l'émetteur et le récepteur sont confondus, ce décalage de fréquence ne contient pas d'information sur la distance à laquelle se trouve le réflecteur. C'est le cas des applications où l'effet Doppler sert à l'étude de la vitesse [3-4]. La technique Doppler pulsé [5-6] a été développée pour pouvoir estimer à la fois la distance et l'effet Doppler. Cette technique est limitée par le faible rapport signal à bruit et le phénomène d'aliasing dus à l'émission pulsée. Pour des applications dans l'air, la distance maximale ainsi mesurée ne dépasse pas $50 \mathrm{~cm}$.

Dans cet article, nous présentons l'étude théorique d'une méthode de localisation spatiale par effet Doppler en émission continue de fréquence constante, et les résultats expérimentaux de la latéralisation d'objet par rapport à l'axe d'émission.

\section{RELATION ENTRE LA FREQUENCE DOPPLER ET LA LOCALISATION 2-D.}

Dans le cas où l'émetteur et le récepteur ne sont pas confondus, la fréquence Doppler que nous notons fD vaut $\mathrm{F}_{0} \frac{\mathrm{V}}{\mathrm{C}}(\cos \theta+\cos \alpha)$ et dépend de $\mathrm{R}$ et $\theta$, Fo étant la fréquence de l'onde émise, $\mathrm{V}$ la vitesse du mouvement, $C$ la célérité des ultrasons, $\theta$ l'angle d'émission et $\alpha$ l'angle de réception.

En supposant la vitesse $\mathrm{V}$ connue, deux équations différentes du type fD $=f(R, \theta)$ suffisent pour estimer $R$ et $\theta$. Ces équations sont obtenues à partir de la disposition des transducteurs d'émission/réception de la figure 1. Elles valent (1), fD1 et fD2 étant respectivement les fréquences Doppler aux récepteurs $\mathrm{R} 1$ et $\mathrm{R} 2$.

Pour connaitre l'évolution des fréquences Doppler ci-dessus dans le demi-espace en face des transducteurs, nous les avons calculées aux deux cas limites $\beta=0$ et $\beta=\pi / 2$. Les résultats ci-dessous sont obtenus dans les conditions

$$
\begin{aligned}
& \text { suivantes: } F_{0}=40 \mathrm{kHz}, \quad C=344 \mathrm{~ms}^{-1} \text { et } \\
& \left\{\begin{array}{l}
\mathrm{fD} 1=\mathrm{f} 0 \frac{\mathrm{V}}{\mathrm{C}}\left(\cos \theta^{\prime}+\frac{\mathrm{R} \cos \theta^{\prime}+\mathrm{asin} \beta}{\sqrt{\mathrm{a}^{2}+\mathrm{R}^{2}+2 \mathrm{aR} \sin \left(\beta-\theta^{\prime}\right)}}\right) \\
\mathrm{fD} 2=\mathrm{f} 0 \frac{\mathrm{V}}{\mathrm{C}}\left(\cos \theta+\frac{\mathrm{R} \cos \theta^{\prime}-\mathrm{asin} \beta}{\sqrt{\mathrm{a}^{2}+\mathrm{R}^{2}-2 \mathrm{aR} \sin \left(\beta-\theta^{\prime}\right)}}\right)
\end{array}\right.
\end{aligned}
$$




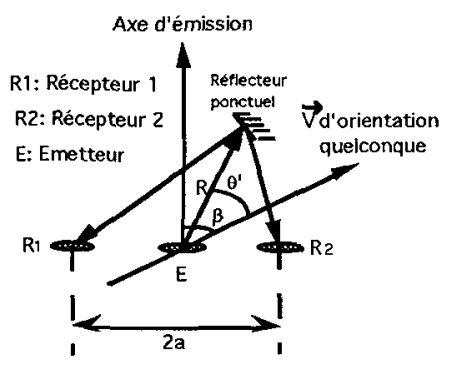

Figure1: Configuration du système de localisation spatiale par effet Doppler avec trois transducteurs.

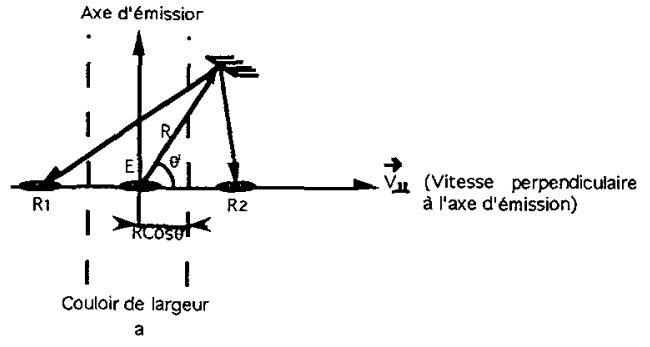

Figure2a: Cas $\beta=\pi / 2$, délimitation de la zone géométrique pour laquelle fol $f D 2<0$.

Pour le cas $\beta=\pi / 2$, on constate qu'il existe une zone autour de l'axe d'émission (figure $2 a$ ) pour laquelle les deux fréquences Doppler sont de signes opposés (figure $2 b)$. Les lieux géométriques $\left(R, \theta^{\prime}\right)$ de cette zone satisfont à la condition:

$$
R \cos \theta^{\prime}<\frac{\mathrm{a}}{2}\left(1+\cos ^{2} \theta\right) \# \frac{\mathrm{a}}{2} \text { lorsque } \frac{\mathrm{a}}{\mathrm{R}}<1
$$
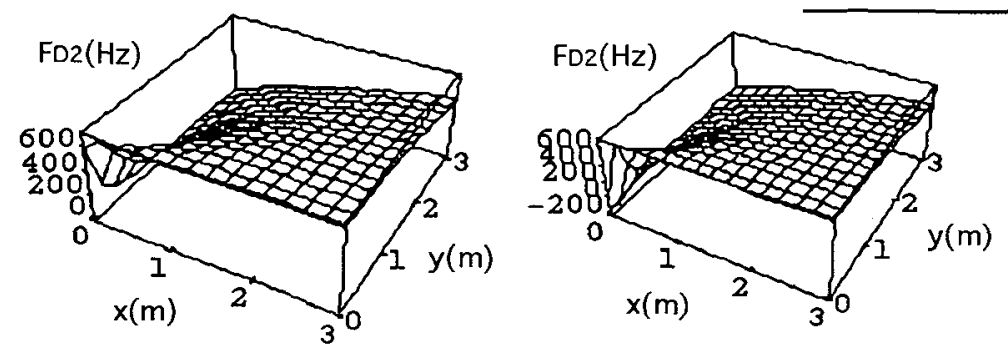

Zoom de la zone pour laquelle les deux fréquences Doppler sont de signes opposés

Figure 2b: Résultats de simulation pour $a=15 \mathrm{~cm}$, cas $\beta=\pi / 2$.

Le cas $\beta=0$ correspond à la situation pour laquelle la vitesse du mouvement est parallèle à l'axe de visée du système de localisation. Compte tenu de cette orientation de la vitesse (figure $3 a$ ), $\theta=-\theta^{\prime}$ et le système d'équations (1) devient (2). Les fréquences fD1 et fD2 sont toujours positives (figure $3 \mathrm{~b}$ ).

(2)

$$
\left\{\begin{array}{l}
\mathrm{fD} 1=\mathrm{f} 0 \frac{\mathrm{V} \cos \theta}{\mathrm{C}}\left(1+\frac{\mathrm{R}}{\sqrt{\mathrm{a}^{2}+\mathrm{R}^{2}+2 \mathrm{aR \operatorname {sin } \theta}}}\right) \\
\mathrm{fD} 2=\mathrm{f} 0 \frac{\mathrm{V} \cos \theta}{\mathrm{C}}\left(1+\frac{\mathrm{R}}{\sqrt{\mathrm{a}^{2}+\mathrm{R}^{2}-2 \mathrm{aR} \sin \theta}}\right)
\end{array}\right.
$$

Quelle que soit l'orientation de la vitesse, les frêquences fD1 et fD2 sont de même ordre de grandeur. Nous nous intéressons au paragraphe suivant à la localisation spatiale 2-D pour le cas $\beta=0$.

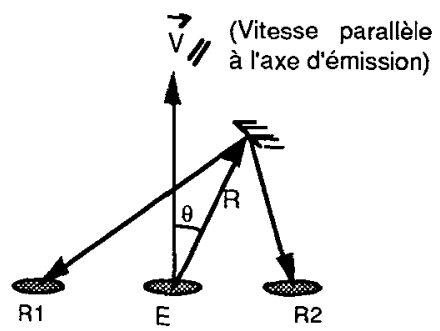

Figure 3a: Cas $\beta=0$. 

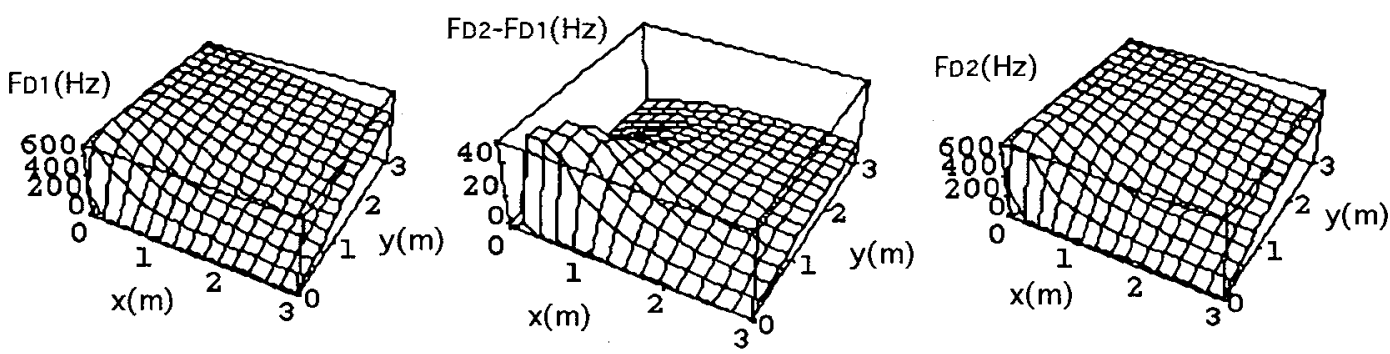

Figure $3 b$ : Résultats de simulation pour $a=15 \mathrm{~cm}$, cas $\beta=0$.

\section{ESTIMATION DES COORDONNEES R, $\theta$.}

Pour $\frac{\mathrm{a}}{\mathrm{R}} \ll 1$ le système d'équations (2) se réduit à:

$$
\left\{\begin{array}{l}
\mathrm{fD} 1=\mathrm{fo} \frac{\mathrm{V}}{\mathrm{C}}\left(1-\frac{\mathrm{a} \sin \theta}{2 \mathrm{R}}\right) \cos \theta=\mathrm{f}_{1}(\mathrm{R}, \theta) \\
\mathrm{fD} 2=\mathrm{f} 0 \frac{\mathrm{V}}{\mathrm{C}}\left(1+\frac{\mathrm{asin} \theta}{2 \mathrm{R}}\right) \cos \theta=\mathrm{f}_{2}(\mathrm{R}, \theta)
\end{array}\right.
$$

Notons:

$\hat{\mathrm{f}}_{\mathrm{D} 1}=\mathrm{f}_{1}(\mathrm{R}, \theta)+\mathrm{e}_{\mathrm{D} 1}$ et $\quad \hat{\mathrm{f}}_{\mathrm{D} 2}=\mathrm{f}_{2}(\mathrm{R}, \theta)+\mathrm{e}_{\mathrm{D} 2}$
L'estimation de $\mathrm{R}$ et $\theta$ par la méthode du maximum de vraisemblance [7] conduit à la solution:

$$
\left\{\begin{array}{l}
\hat{\theta}=\cos ^{-1}\left(\frac{C}{4 V f o}\left(\hat{\mathrm{f}} \mathrm{D} 1+\hat{\mathrm{f}}_{\mathrm{D} 2}\right)\right) \\
\hat{\mathrm{R}}=\frac{\mathrm{a} \sqrt{1-\cos ^{2} \hat{\theta}}\left(\hat{\mathrm{f}}_{\mathrm{D} 1}+\hat{\mathrm{f}}_{\mathrm{D} 2}\right)}{2\left(\hat{\mathrm{f}}_{\mathrm{D} 1}-\hat{\mathrm{f}}_{\mathrm{D} 2}\right)}
\end{array}\right.
$$

Le signe de $\hat{\theta}$ est obtenu en comparant $\hat{\mathrm{f}}_{\mathrm{D} 1}$ à $\hat{\mathrm{f}}_{\mathrm{D} 2}$, puisque le décalage de fréquence Doppler est toujours plus important au récepteur du côté duquel se trouve le réflecteur. Il indique la localisation d'objet à gauche ou à droite de l'axe d'émission, ce que nous désignons ici par le terme latéralisation d'objet (cf. § 4).

La meilleure précision d'estimation des coordonnées $(\hat{\mathrm{R}}, \hat{\theta})$ est obtenue aux bornes de CramerRao [8] dont le calcul montre qu'elles dépendent de V, C, R, $\theta$ et a. La précision de mesure de $\hat{\theta}$ est meilleure que celle de $\hat{R}$, qui dépend de l'inverse de la différence de fréquences (fD2-fDI).

Le système électronique schématisé à la figure 5 met en œuvre le traitement de signal défini dans ce paragraphe. Nous avons vérifié expérimentalement l'existence des signaux Doppler de fréquences $(f D 2+f D 1)$ et $(f D 2-f D 1)$.

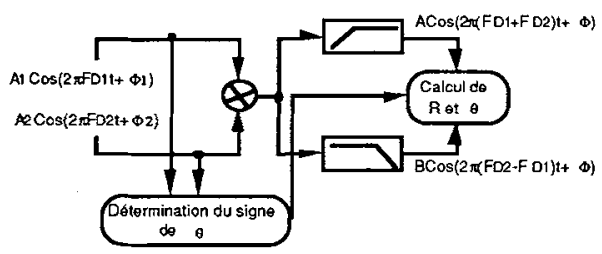

Figure 5: Principe du traitement des signaux Doppler pour la localisation spatiale, cas du système E, R1, R2 (figure $3 a$ ).

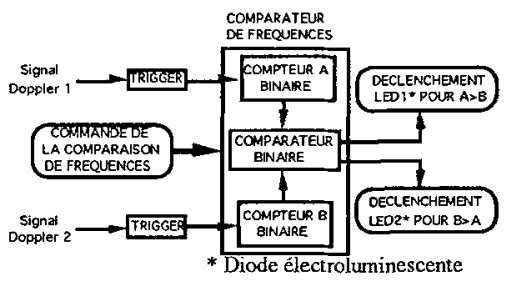

Figure 6a: Système Doppler à émission continue utilisé pour la latéralisation d'objet.

\section{VERIFICATION EXPERIMENTALE DE LA LATERALISATION D'OBJET}

\subsection{Description du système Doppler}

Ce système est composé d'un émetteur et de deux récepteurs d'angle de directivité en émission à 
$-6 \mathrm{~dB}$ de $35^{\circ}$, fonctionnant à $40 \mathrm{kHz}$ et disposés comme l'indique le schéma de la figure 3 . Les signaux Doppler détectés aux deux récepteurs sont envoyés au comparateur de fréquences (figure 6a) dont le résultat (déclenchement de l'une des diodes électroluminescentes) indique le signal de fréquence la plus élevée, donc le signe de l'angle $\theta$ et la localisation de l'objet détecté par rapport à l'axe d'émission.

\subsection{Résultats et interprétation}

La comparaison des deux fréquences (commande externe) dure $500 \mathrm{~ms}$, ce qui correspond à une résolution d'analyse en fréquence supérieure à $2 \mathrm{~Hz}$. Les mesures présentées à la figure $6 \mathrm{~b}$ ont été faites par pas de $0.5 \mathrm{~cm}$ en (x) et par pas de $25 \mathrm{~cm}$ en (y), avec un objet cylindrique de diamètre $4 \mathrm{~cm}$ animé d'un mouvement de vitesse $1 \mathrm{~ms}^{-1}$. Chaque point de mesure est répété vingt fois; la localisation certaine de l'objet n'est décidée que lorsque la diode est déclenchée plus de dix fois, c'est l'espace situé en dessous de la courbe d'ajustement des points expérimentaux. L'espace situé au dessus de cette courbe est la zone d'incertitude de la latéralisation, due à la résolution du comparateur de fréquences qui ne permet pas de différencier les deux fréquences Doppler. Il correspond à l'espace théorique délimité par $(\mathrm{fD2}-\mathrm{fD} 1)<3 \mathrm{~Hz}$.

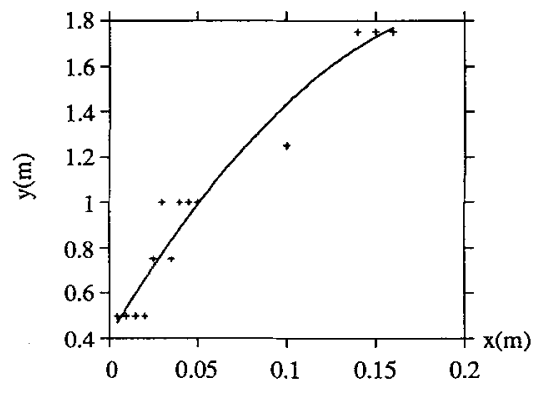

Figure 6b: Résultats expérimentaux de la latéralisation d'un objet cylindrique de $4 \mathrm{~cm}$ de diamètre. L'émetteur est situé en $(0,0), a=15 \mathrm{~cm}$.

\section{CONCLUSION}

La grande sensibilité du signal Doppler permet d'envisager la détection d'environnement dans l'air à des distances plus importantes. Une méthode de localisation spatiale en émission continue de fréquence constante basée sur l'effet Doppler a été présentée. Sur le plan théorique, il s'agit d'une méthode de localisation inédite, au delà de la longueur d'onde, qui ne nécessite ni référence temporelle (comme en émission pulsée) ni référence fréquentielle (comme en émission modulée en fréquence). Les résultats expérimentaux de la latéralisation d'objet ont confirmé une étape et un cas particulier de cette méthode. Toutefois, des limitations sont à souligner: $i$ ) la mesure de distance impossible sur l'axe d'émission, car $\mathrm{fD} 1=\mathrm{fD} 2, i i)$ la connaissance de la vitesse du mouvement, $i i i$ ) le compromis entre le temps d'analyse en fréquence et la précision de la solution.

\section{REFERENCES}

[1] Toman K., Christian Doppler and the Doppler effect, Eos, Vol. 65, 1984, 1193-1194.

[2] Censor D., Acoustical Doppler analysis-Is a valid method?, J. Acoust. Soc. Am., Vol. 83, 1987, 12231230.

[3] Satomura S., Ultrasonic Doppler method for the inspection of cardiac functions, J. Acoust. Soc. Am., Vol. 29, 1957, 1181-11185.

[4] Pourcelot L., Etude et réalisation d'un débimètre sanguin à effet Doppler utilisable en télé-mesure, Thèse de docteur-ingénieur, Université de Lyon, 1967.

[5] Baker D.W., Pulsed ultrasonic blood flow sensing, IEEE trans. So, Ultrason, Vol. 17, 170-185, 1970.

[6] Peronneau P.A. et al., Theoretical and pratical aspects of pulsed Doppler flowmetry: real-time application to the measure of instantaneous velocity profiles in vitro and in vivo, In: Cardiovascular application of ultrasound, Ed. Reneman R.S., Amsterdam, 1974, 66-84.

[7] Kay S.M., Modern spectral estimation - Theory \& Application, Prentice-Hall, 1988. 